\title{
Council of Lloyd's
}

\section{Chairman \\ Murray Lawrence \\ Deputy Chairmen \\ David Coleridge \\ Colin Murray}

\section{Deputy Chairman and}

Chief Executive

Alan Lord, CB

\section{Working Members \\ John Greig \\ Richard Hazell \\ Anthony Hines \\ Gordon Hutton \\ Alan Jackson}

Stephen Merrett

Sir Peter Miller

David Rowland

Michael Wade

\section{Nominated Members}

Patrick Egan

Sir Maurice Hodgson

Sir Jeremy Morse

Matthew Patient

Brian Pomeroy

Mark Sheldon

David Walker

External Members

Mary Archer

Sir Nicholas Bonsor, Bt, MP

Sir Alcon Copisarow, DSc

Mark Farrer

The Rt Hon Lord Kimball, DL

Eddie Kulukundis

Sir Gerald Peat

The Rt Hon. The Lord

Rees, QC
Retirement

date

1991

1991

1992

- $\quad$ Nominated Member

1992

1989

1992

1989

1990

1990

1989

1990

1991

1991

1991

1990

1989

1989

1991

1992

1991

1990

1991

1990

1989

1992

1989

\section{Greig Fester Ltd}

Joseph W Hobbs \& Co.,

Merrett Syndicates Ltd

Horace Holman \& Co Ltd

Main Lloyd's business connection

Murray Lawrence \& Partners

R W Sturge \& Co. Ltd

R J Kiln \& Co.,

Spur Kiln Underwriting Agency Ltd,

Dann Kiln Underwriting Agency Ltd

See p. $\mathrm{xx}$

Three Quays Underwriting Management Ltd

Crowe Underwriting Agencies Ltd

G W Hutton \& Co. (Underwriting Agency) Ltd

Robert Bradford Underwriting Ltd,

Alan Jackson Underwriting Agency Ltd

Thos R Miller \& Son Underwriting Agency Ltd

Sedgwick Lloyd's Underwriting Agency Ltd

Chairman, Securities \& Investment Board 